\title{
Solution structure of the soluble domain of the NfeD protein YuaF from Bacillus subtilis
}

\author{
Christina A. Walker • Markus Hinderhofer • \\ David J. Witte · Winfried Boos · Heiko M. Möller
}

\begin{abstract}
The transmembrane protein YuaF from $B$. subtilis is a member of the NfeD-like clan with a potential role in maintaining membrane integrity during conditions of cellular stress. $n f e D$-genes are primarily found in highly conserved operon structures together with the gene of another membrane protein belonging to the SPFH superfamily, in this case YuaG. This strongly suggests a functional if not physical interaction between YuaF and YuaG. Secondary structure predictions of NfeD proteins that accompany SPFH proteins all indicate a high content of $\beta$-sheets in the C-terminal domains indicating a conserved core structure despite very low homology at the level of primary structure. Here we report the high-resolution solution structure of YuaF's soluble C-terminal domain derived from NMR data (sYuaF, residues 97-174 of full-length YuaF). Full backbone and side chain assignments of $\mathrm{s} Y u a F$ were obtained from triple-resonance spectra. The structure was determined from distance restraints derived from $3 D$ NOESY spectra collected at $600 \mathrm{MHz}$ and $800 \mathrm{MHz}$, together with $\varphi, \psi$, and $\chi_{1}$ torsion angle restraints based on the analysis of ${ }^{1} \mathrm{H}^{\mathrm{N}},{ }^{15} \mathrm{~N},{ }^{1} \mathrm{H}^{\alpha}$,
\end{abstract}

C. A. Walker and M. Hinderhofer contributed equally to this work. Residue numbering: For the remainder of this publication we use a numbering scheme according to the sequence of our protein construct sYuaF (soluble YuaF). Residues Ser $^{7}$ to $\mathrm{Ile}^{84}$ of $\mathrm{sYuaF}$ correspond to amino acids 97 to 174 of full-length YuaF. The first six residues, Gly $^{1}$ to Glu ${ }^{6}$, originate from introducing the thrombin cleavage site.

C. A. Walker - D. J. Witte - H. M. Möller (区)

Department of Chemistry, University of Konstanz,

Universitätsstraße 10, 78457 Konstanz, Germany

e-mail: heiko.moeller@uni-konstanz.de

M. Hinderhofer W. Boos

Department of Biology, University of Konstanz,

Universitätsstraße 10, 78457 Konstanz, Germany
${ }^{13} \mathrm{C}^{\alpha},{ }^{13} \mathrm{CO}$, and ${ }^{13} \mathrm{C}^{\beta}$ chemical shifts, and HNHA, HNHB and HACAHB-COSY spectra. Structures were calculated using CYANA 2.0 and refined in AMBER 8. SYuaF is composed of an extended $N$-terminal $\alpha$-helix and a $\beta$-barrel formed by five $\beta$-strands. This $\beta$-sheet core structure is well known from the diverse class of $\mathrm{OB}$-fold proteins and can also be found in the distantly related NfeD protein $\mathrm{Ph} 0471$ from the archaeon $P$. horikoshii. Despite significant differences of their amino acid sequences the structural homology of these proteins suggests a conserved function of SPFH-associated NfeD proteins.

Keywords YuaF - Bacillus subtilis - Cellular stress . OB-fold · Reggie/flotillin - SPFH superfamily

\section{Biological context}

YuaF from B. subtilis strain Marburg 168 is a transmembrane protein encoded by the first gene of the yuaFGI operon. Expression of YuaF is $\sigma^{w}$-dependent suggesting a role in the response to cell envelope stress caused e.g. by alkaline shock or presence of toxic peptides (Wiegert et al. 2001). Based on its primary structure, YuaF belongs to the NfeD-like clan (Bateman et al. 2004). The name NfeD was introduced by Soto et al. to designate a couple of proteins which are involved in the nodulation efficiency and competitiveness (nfe) of Sinorhizobium meliloti (Soto et al. 1994), however, in the meantime many proteins have been identified that carry the NfeD signature but are unrelated to nodulation efficiency. Many nfeD-genes are found in operons which also contain the gene of an SPFH superfamily protein (Tavernarakis et al. 1999), in our case the reggie/flotillin homolog YuaG, the eukaryotic relatives of 
which are involved in membrane organization and cellular regeneration.

The co-expression and co-regulation of yuaF and yuaG as a consequence of their occurrence in the same operon structure, and the potential link between cellular regeneration in eukaryotes and response to cellular stress in prokaryotes is indicative for a functional connection between YuaF and YuaG in particular, and between NfeD and SPFH proteins in general.

The high-resolution NMR structure of the soluble C-terminal domain of YuaF is our first step on the way to elucidate the structural and functional role of NfeD-proteins and their interplay with SPFH proteins.

\section{Methods and results}

\section{Expression and purification}

The region of $y u a F$ which codes for the C-terminal, soluble part of YuaF, residues 97-174 of the full-length protein, was amplified with primers F_XhoI_up (ATCTCGAGTCATC TGCAGAAGAATCATTGG) and F_HindIIId (TAAA GCTTGTTAAATGGGTTCATGCGGAG). The obtained PCR product ( $254 \mathrm{bp}$ ) was cleaved with XhoI and HindIII and inserted into pHIHO1422. The fragment of yuaF was thereby fused in frame to the $3^{\prime}$-end of the malE gene. pHIHO1422 is a pmal-c $2 x$ derivate of our lab (unpublished data) and harbours a thrombin cleavage site to cut off the MalE protein from the MalE-s YuaF construct. The inserted sequence has been confirmed by sequencing and the plasmid was named pHIHO1421.

Escherichia coli BL21 were transformed with pHIHO1421 and selected on ampicillin $(100 \mu \mathrm{g} / \mathrm{ml}) / \mathrm{LB}$ agar plates. The overnight culture grew up in $20 \mathrm{ml} \mathrm{LB} / \mathrm{ampi}-$ cillin and was used to inoculate $1 \mathrm{~L} \mathrm{M9}$ minimal medium, supplemented with $0.1 \%{ }^{15} \mathrm{NH}_{4} \mathrm{Cl}$ and $0.4 \%{ }^{13} \mathrm{C}_{6}$-D-glucose as sole nitrogen and carbon sources, respectively. Additionally, ampicillin and BME vitamins solution (Sigma Aldrich) were added. Six hours after incubation at $37^{\circ} \mathrm{C}$ expression of MalE-sYuaF was induced with $1 \mathrm{mM}$ isopropyl- $\beta$-D-thiogalactoside and cells were harvested after $12 \mathrm{~h}$.

The bacterial pellets were resuspended in buffer $A$ (50 mM Tris, pH 7.5 and $300 \mathrm{mM} \mathrm{NaCl}$ ) and ruptured by passage through a French pressure cell. After centrifugation at $100,000 \mathrm{~g}$ for $1 \mathrm{~h}$ at $4^{\circ} \mathrm{C}$ the supernatant was loaded on a column packed with $\sim 15 \mathrm{ml}$ Amylose resin (New England Biolabs). Nearly pure fusion protein was eluted with buffer B (50 mM Tris, pH 7.5, $300 \mathrm{mM} \mathrm{NaCl}, 10 \mathrm{mM}$ maltose). The fusion protein was incubated with thrombin protease, and completeness of digestion was verified by SDS page. Maltose binding protein $(44.5 \mathrm{kDa})$ and $\mathrm{s} Y u a F$
$(8.8 \mathrm{kDa})$ were separated by gel filtration, using a HiLoad 16/60 Superdex75 prep grade column (Amersham) and buffer A. sYuaF was concentrated with Amicon Ultra 4 (Millipore) to $1 \mathrm{mM}$. Our final yield of purified sYuaF was $11 \mathrm{mg} / \mathrm{l}$ of culture medium.

\section{NMR spectroscopy}

NMR experiments with the purpose of backbone, aliphatic and aromatic side chain resonance assignment were performed on a $0.9 \mathrm{mM}^{13} \mathrm{C},{ }^{15} \mathrm{~N}$-labelled sample in $\mathrm{H}_{2} \mathrm{O} / \mathrm{D}_{2} \mathrm{O}$ 95:5 (v:v) or in pure $\mathrm{D}_{2} \mathrm{O}$ and a $1 \mathrm{mM}^{15} \mathrm{~N}$-labelled sample in $\mathrm{H}_{2} \mathrm{O} / \mathrm{D}_{2} \mathrm{O} 95: 5$ (v:v). All sample solutions were buffered at $\mathrm{pH}=7.5$ with $50 \mathrm{mM}$ Tris, and contained $300 \mathrm{mM}$ $\mathrm{NaCl}$ and $4 \mathrm{mM} \mathrm{NaN}$. NMR spectra were recorded at $280 \mathrm{~K}$ on a $600 \mathrm{MHz}$ Bruker Avance DRX spectrometer equipped with a TXI- $\left({ }^{1} \mathrm{H},{ }^{13} \mathrm{C},{ }^{15} \mathrm{~N}\right)$ triple resonance probe, and, in case of the ${ }^{13} \mathrm{C}$ NOESY HSQC, on a $800 \mathrm{MHz}$ Bruker Avance III spectrometer equipped with a TCI- $\left({ }^{1} \mathrm{H}\right.$, ${ }^{13} \mathrm{C}$, ${ }^{15} \mathrm{~N}$ ) triple resonance cryogenic probe. ${ }^{1} \mathrm{H},{ }^{13} \mathrm{C}$, and ${ }^{15} \mathrm{~N}$ chemicals shifts were referenced using the values for reference correction obtained with the PREDITOR subprogram REFCOR (Berjanskii et al. 2006). NMR data were processed using XWINNMR/TOPSPIN (Bruker Biospin, Karlsruhe, Germany) or NMRPipe (Delaglio et al. 1995). For data analysis the program CARA (Keller 2004) was used. For sequential backbone assignment the following spectra were used: $\left[{ }^{1} \mathrm{H},{ }^{15} \mathrm{~N}\right]-\mathrm{HSQC}, \mathrm{HNCO}$, $\mathrm{HN}(\mathrm{CA}) \mathrm{CO}, \mathrm{HNCACB}, \mathrm{CBCA}(\mathrm{CO}) \mathrm{NH}$ supported by ${ }^{15} \mathrm{~N}$ TOCSY and NOESY HSQC. Aliphatic ${ }^{1} \mathbf{H}$ and ${ }^{13} \mathrm{C}$ side chain assignments are based on constant-time $\left[{ }^{1} \mathrm{H},{ }^{13} \mathrm{C}\right]-$ $\mathrm{HSQC}, \mathrm{H}(\mathrm{C}) \mathrm{CH}-\mathrm{COSY}, \mathrm{H}(\mathrm{C}) \mathrm{CH}-\mathrm{TOCSY}$ and $(\mathrm{H}) \mathrm{CCH}-$ TOCSY experiments in conjunction with a ${ }^{13} \mathrm{C}$ NOESY HSQC. A constant-time $\left[{ }^{1} \mathrm{H},{ }^{13} \mathrm{C}\right]-\mathrm{HSQC}$ centered to the aromatic region and optimized for suppressing the aromatic CC-coupling in addition to (HB) $\mathrm{CB}$ (CGCD)HD and (HB) $\mathrm{CB}$ (CGCDCE)HE spectra, and a ${ }^{13} \mathrm{C}$ NOESY HSQC of the aromatic region provided unambiguous assignments of the aromatic side chains except for the chemical shifts of $\mathrm{H}^{i 1}$ of $\mathrm{His}^{3}$ and $\mathrm{His}^{81}$. The assignment of the $\mathrm{NH}_{2}$-groups of the side chains of Asn and Gln was achieved with a combination of the $\left[{ }^{1} \mathrm{H},{ }^{15} \mathrm{~N}\right]-\mathrm{HSQC}, \mathrm{CBCA}(\mathrm{CO}) \mathrm{NH}$ and ${ }^{15} \mathrm{~N}$ HSQC NOESY. Finally, two of the three $\mathrm{N}^{t} \mathrm{H}^{2}$ - and $\mathrm{C}^{\zeta}-$ resonances of the arginine guanidino groups ( $\operatorname{Arg}^{16}$ and $\mathrm{Arg}^{2 \mathrm{I}}$ ) could be assigned by combining information from $\left[{ }^{1} \mathrm{H},{ }^{15} \mathrm{~N}\right]-\mathrm{HSQC}, \mathrm{HNCO}$ and HNCACB.

With the exception of the ${ }^{1} \mathrm{H}^{\mathrm{N}},{ }^{15} \mathrm{~N}$, and $\mathrm{C}$ ' chemical shifts of the residues $\mathrm{Gly}^{1}, \mathrm{Ser}^{2}, \mathrm{His}^{3}$ and $\mathrm{Asn}^{73}$, and the ${ }^{15} \mathrm{~N}$ chemical shifts of $\mathrm{Pro}^{32}, \mathrm{Pro}^{80}$, and Pro ${ }^{83}$, complete backbone assignments of all residues were obtained. ${ }^{1} \mathrm{H}$ chemical shifts were obtained for $90.5 \%$ of the side chain $\mathrm{CH}_{n}$ moieties $(91.4 \%$ of aliphatic sidechain protons and $78.6 \%$ of aromatic side chains) and $91.8 \%$ of the sidechain 
${ }^{13} \mathrm{C}$ resonances $(93.7 \%$ of the aliphatic and $73.3 \%$ of the aromatic carbon chemical shifts). Complete assignments of the $\mathrm{N}^{\gamma} \mathrm{H}_{2}$ and $\mathrm{N}^{\delta} \mathrm{H}_{2}$ of the three Asn and Gln residues, respectively, as well as assignments of the aromatic side chains of the two Tyr and Phe amino acids of sYuaF were obtained. The ${ }^{15} \mathrm{~N}$-HSQC spectrum reveals an additional set of weak cross peaks (intensity $<10 \%$ relative to other HN cross peaks) which belong to $\mathrm{Glu}^{82}$ and $\mathrm{Il}^{84}$ and which arise from cis-trans isomerism of $\mathrm{Pro}^{83}$. According to chemical shifts of $\mathrm{C}^{\beta}$ and $\mathrm{C}^{\gamma}, \mathrm{Pro}^{83}$ is trans-configured in the dominant isomer (Schubert et al. 2002). This is also supported by the pattern of local NOEs. Figure 1 shows the assigned ${ }^{15} \mathrm{~N}$ HSQC spectrum of sYuaF that resulted from the assignment procedure described above. The ${ }^{1} \mathrm{H},{ }^{13} \mathrm{C}$ and ${ }^{15} \mathrm{~N}$ chemical shift assignments have been deposited in the BioMagResBank database with accession code 15475 .

\section{Structure determination}

Distance constraints were derived from NOE cross peak volumes observed in a $600 \mathrm{MHz}{ }^{15} \mathrm{~N}$-separated NOESY HSQC spectrum, a $600 \mathrm{MHz}{ }^{13} \mathrm{C}$-separated NOESY HSQC of the aromatic region, and a $800 \mathrm{MHz}{ }^{13} \mathrm{C}$-separated NOESY HSQC of the aliphatic region with mixing times of
$100 \mathrm{~ms}, 120 \mathrm{~ms}$, and $90 \mathrm{~ms}$, respectively. Backbone torsion angle restraints were included based on the analysis of ${ }^{\prime} \mathrm{H}^{\mathrm{N}}$, ${ }^{15} \mathrm{~N},{ }^{1} \mathrm{H}^{\alpha},{ }^{13} \mathrm{C}^{\alpha},{ }^{13} \mathrm{CO}$, and ${ }^{13} \mathrm{C}^{\beta}$ chemical shifts using the program PREDITOR (Berjanskii et al. 2006) and supported by ${ }^{3} \mathrm{~J}_{\mathrm{HNH} z}$ coupling constants measured with an HNHA spectrum, and the analysis of local NOEs. The solely chemical shift-based $\psi$ angle restraints were treated conservatively and applied in regions of confirmed secondary structure only. After close inspection of the NOE network $\varphi$ angle constraints in accordance with ${ }^{3} \mathrm{~J}_{\mathrm{HNH} \alpha}$ were also applied in loop regions $\left(\mathrm{Ser}^{61}, \mathrm{Tyr}^{62}, \mathrm{Asn}^{72}\right.$, and $\mathrm{Val}^{75}$ ). In the absence of more specific information the $\varphi$ angle of lle and $\mathrm{Val}$ residues was constrained to $-180-0^{\circ}$ taking into account the restricted conformational freedom of these bulky amino acids. Additionally, $\chi_{1}$ torsion angle restraints derived from HNHB and HACAHB-COSY spectra were employed. Stereospecific assignments were made according to coupling constants determined from HNHA, HNHB and HACAHBCOSY spectra and the local NOE pattern. Automated iterative NOE assignment and structure calculation was carried out with the CANDID/NOEASSIGN module of CYANA 2.0 (Güntert et al. 1997; Herrmann et al. 2002) in conjunction with manual inspection and verification of assigned and rejected NOEs. A separate chemical shift list was used for
Fig. $1\left[{ }^{1} \mathrm{H},{ }^{15} \mathrm{~N}\right]-\mathrm{HSQC}$ of $0.9 \mathrm{mM}{ }^{13} \mathrm{C},{ }^{15} \mathrm{~N}$-labelled sYuaF in $50 \mathrm{mM}$ Tris buffer, 300 $\mathrm{mM} \mathrm{NaCl}, \mathrm{pH} 7.54 \mathrm{mM} \mathrm{NaN}_{3}$ in $\mathrm{H}_{2} \mathrm{O} / \mathrm{D}_{2} \mathrm{O} 95 / 5$ (v:v) recorded at $600 \mathrm{MHz}$ and $7^{\circ} \mathrm{C}$. The assignments are indicated with one letter code and residue number. Amide ${ }^{\delta} \mathrm{NH}_{2}$ of Asn and " $\mathrm{NH}_{2}$ of Gln are connected by lines and denoted as "sc". The Arg ${ }^{8} \mathrm{NH}$ are also indicated as "sc". Both ${ }^{E} \mathrm{NH}$ of $\mathrm{Arg}^{16}$ and $\operatorname{Arg}^{21}$ are folded in the ${ }^{15} \mathrm{~N}$ dimension

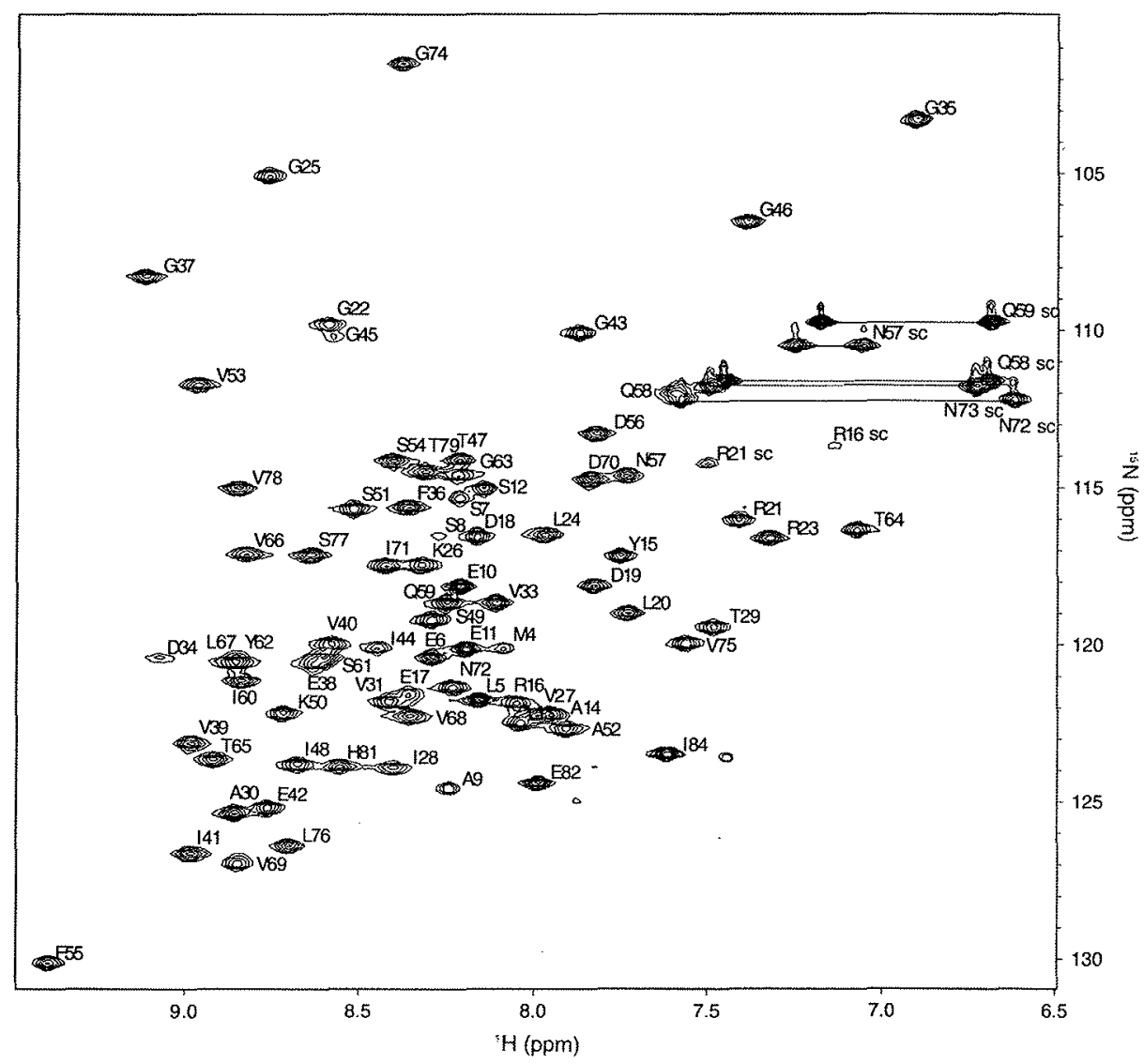


each NOESY spectrum to account for slight variations in sample conditions. The standard protocol of CYANA 2.0 was used with seven cycles of combined automated NOE assignment and structure calculation of 100 conformers in each cycle.

The final set of restraints was obtained by adjusting upper distance bounds to account for spectral overlap and by manually resolving ambiguities. It consisted of 1526 distance and 97 dihedral angle restraints which were used to calculate 400 conformers by torsion angle dynamics simulated annealing in CYANA. The 100 structures with lowest target function served as starting structures for refinement in AMBER 8 (Case et al. 2004). Structures were minimized, subjected to one round of simulated annealing in vacuum with charges reduced to $20 \%$ of their original value, followed by simulated annealing with full charges in a generalized Born solvation model (Bashford and Case 2000) and subsequent energy minimization. All calculations were carried out on Intel Xeon processors with eight processor cores running under CentOS Linux (Kernel 2.6.24.2). An ensemble of 20 conformers with lowest combined restraint violations was selected for further analysis. Input data and structure calculation statistics are summarized in Table 1 . The quality of the structural ensemble was evaluated with PROCHECK-NMR (Laskowski et al. 1996) and visualized with MOLMOL (Koradi et al. 1996). The 3D coordinates and experimentally derived restraints of $s Y u a F$ have been deposited in the RCSB Protein Data Bank with accession code 2 K14.

Figure 2a shows a stereo representation of the final ensemble of 20 structures. The high number of NOEs (1526 in total, 522 long-range) leads to an excellent definition of the structural core of $s$ YuaF with an r.m.s.d. of $0.57 \AA$ when superimposing heavy atoms from residue $\mathrm{Glu}^{17}$ to $\mathrm{Glu}^{82}$. The vast majority of residues (99.9\%) populate the most favourable and additionally allowed regions of $\varphi / \psi$-space.

In Fig. $2 b$ the lowest energy conformer is shown in ribbon representation. The protein $\mathrm{S} Y u a \mathrm{~F}$ comprises of an extended $N$-terminal $\alpha$-helix followed by a five-stranded $\beta$-barrel that arranges in a topology reminiscent of the oligonucleotide/oligosaccharide-binding-(OB)-protein fold (Murzin 1993).

The five $\beta$-strands form a closed $\beta$-barrel structure with a 1-2-3-5-4-1 topology characteristic for the OB-fold with secondary structure elements ranging from $\mathrm{Leu}^{24}$ to $\mathrm{Thr}^{29}$ $(\beta 1), \mathrm{Phe}^{36}$ to $\mathrm{Ile}^{41}(\beta 2), \mathrm{Lys}^{50}$ to $\operatorname{Ser}^{54}(\beta 3), \mathrm{Thr}^{65}$ to $\mathrm{Asn}^{72}$ $(\beta 4)$ and $\mathrm{Val}^{75}$ to $\mathrm{Thr}^{79}(\beta 5)$. $\beta 1$ pairs in an anti-parallel fashion with $\beta 2$ and $\beta 4$, however, the anti-parallel section between $\beta 1$ and $\beta 4$ is rather short compared to canonical OB-fold proteins. The same holds true for the anti-parallel arrangement between $\beta 2$ and $\beta 3$, instead, $\beta 3$ forms an
Table 1 Structural statistics of the final ensemble of 20 conformers of the C-terminal domain of YuaF from $B$, subtilis after refinement in AMBER 8

\begin{tabular}{|c|c|}
\hline \multicolumn{2}{|l|}{ (A) NMR restraints } \\
\hline Total unambiguous distance restraints & 1526 \\
\hline Intraresidue $(\mathbf{i}, \mathbf{i})$ & 379 \\
\hline Sequential $(i \mathrm{i}-\mathrm{j} l=1)$ & 411 \\
\hline Medium-range $(1<|\mathrm{i}-\mathrm{j}|<5)$ & 214 \\
\hline Long-range $(|\mathrm{i}-\mathrm{j}|>4)$ & 522 \\
\hline Total dihedral angle restraints & 97 \\
\hline$\varphi$ & 49 \\
\hline$\psi$ & 34 \\
\hline$x_{1}$ & 14 \\
\hline \multicolumn{2}{|l|}{ (B) Ensemble statistics (20 structures) } \\
\hline \multicolumn{2}{|l|}{ Violation analysis } \\
\hline Maximum distance violation $(\AA)$ & 0.17 \\
\hline Maximum dihedral angle violation (deg.) & 4.83 \\
\hline \multicolumn{2}{|l|}{ Energies } \\
\hline Mean restraint violation energy $\left(\mathrm{kcal} \mathrm{mol}^{-1}\right)$ & $5.3 \pm 0.2$ \\
\hline Mean AMBER energy (kcal $\mathrm{mol}^{-1}$ ) & $-2032 \pm 6$ \\
\hline \multicolumn{2}{|l|}{ Mean deviation from ideal covalent geometry } \\
\hline Bond lengths $(\AA)$ & 0.01 \\
\hline Bond angles (deg.) & $2.06 \pm 0.02$ \\
\hline \multicolumn{2}{|l|}{ RMSD from mean structure } \\
\hline \multirow[t]{2}{*}{ Backbone heavy atoms $\left(N, C^{\alpha}, C^{\prime}, O\right)$} & 0.58 (all residues) \\
\hline & $\begin{array}{l}0.21 \text { (residues } \\
17-82 \text { ) }\end{array}$ \\
\hline \multirow[t]{2}{*}{ All heavy atoms } & 0.93 (all residues) \\
\hline & $\begin{array}{l}0.57 \text { (residues } \\
17-82 \text { ) }\end{array}$ \\
\hline \multicolumn{2}{|l|}{ Ramachandran plot } \\
\hline Most-favorable regions $(\%)$ & 87.4 \\
\hline Additionally allowed regions (\%) & 12.5 \\
\hline Generously allowed regions (\%) & 0.1 \\
\hline Disallowed regions $(\%)$ & 0.0 \\
\hline
\end{tabular}

unusually long parallel $\beta$-sheet with $\beta 5$ that itself forms contacts with $\beta 4$ again in anti-parallel fashion.

$\beta 1$ and $\beta 2$ are connected by a $\beta$-hairpin of residues $\operatorname{Pro}^{32}$ to $\mathrm{Gly}^{35}$. Residues $\mathrm{Glu}^{42}$ to $\mathrm{Ser}^{49}$ connecting $\beta 2$ and $\beta 3$ adopt an irregular structure; likewise, the long connecting sequence between $\mathrm{Ser}^{54}$ of $\beta 3$ and $\mathrm{Thr}^{65}$ of $\beta 4$ does not show a regular secondary structure; an $\alpha$-helix, characteristic for the canonical OB-fold at this position, is absent in the structure of $\mathrm{sYuaF}$. The last two strands, $\beta 4$ and $\beta 5$, are again connected by a $\beta$-hairpin of residues $\mathrm{Asn}^{72}$ to $\mathrm{Val}^{75}$.

Interestingly, the hydrophobic core is exclusively formed by packing of aliphatic side chains of residues $\mathrm{Leu}^{20}, \mathrm{Val}^{27}, \mathrm{Val}^{31}, \mathrm{Val}^{39}, \mathrm{Ile}^{41}, \mathrm{Ala}^{52}, \mathrm{Ile}^{60}, \mathrm{Val}^{66}, \mathrm{Val}^{68}$, $\mathrm{Ile}^{71}$, $\mathrm{Leu}^{76}$, and $\mathrm{Val}^{78}$. All aromatic side chains are located on the surface of sYuaF (Fig. 3a) and show, in agreement 
Fig. 2 The high resolution NMR structure of C-terminal domain of YuaF from $B$. subtilis. (a) Stereo representation of the ensemble of 20 structures with lowest constraint violations. Backbone atoms are grey, side chain heavy atoms are blue. The ensemble has been fitted to heavy atoms of the structural core encompassing residues $\mathrm{Glu}^{17}$ $\mathrm{Glu}^{82}$. (b) Stereo view of the ribbon diagram of the conformer with lowest constraint energy
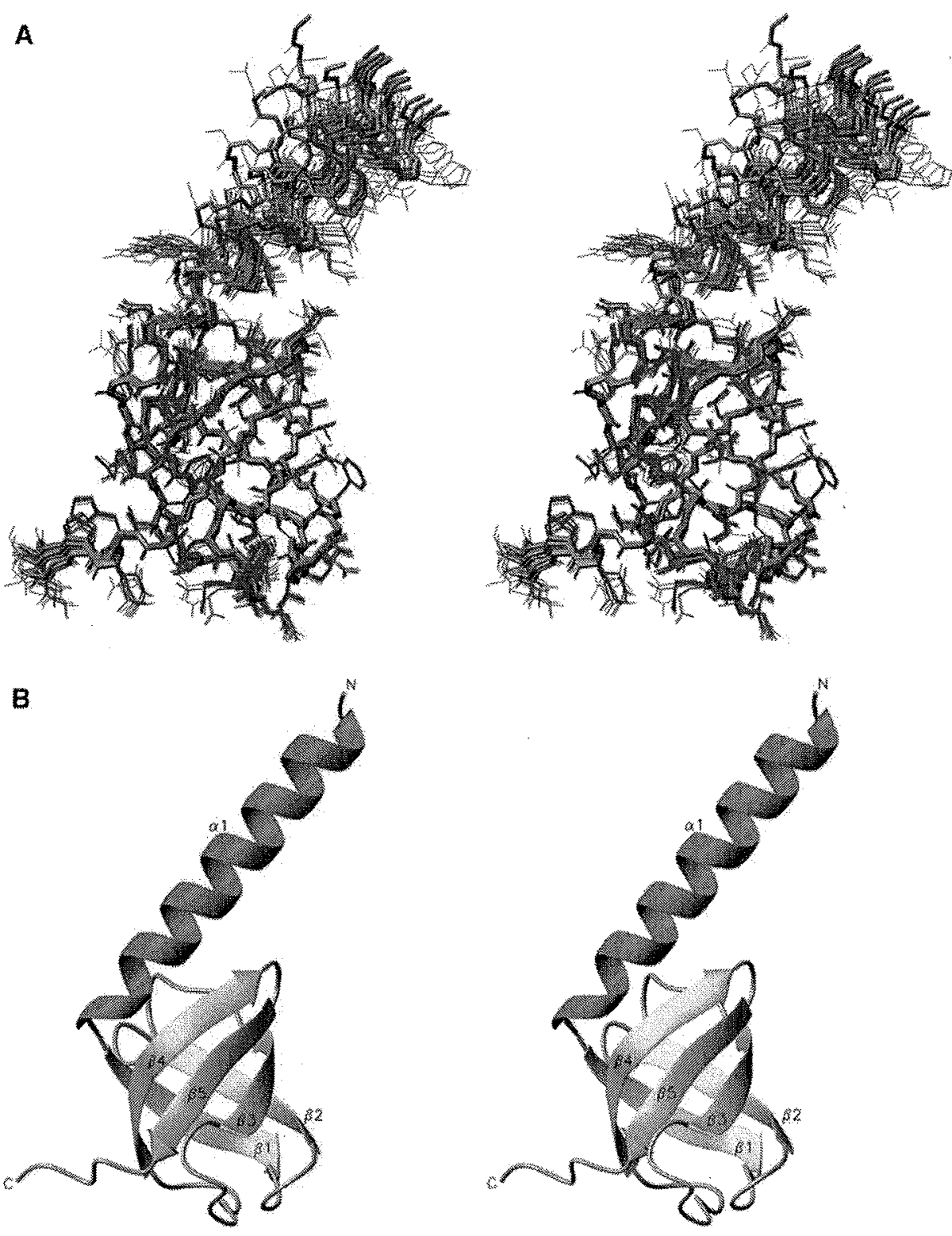

with the structure, only a limited number of NOE contacts to other solvent accessible residues.

\section{Backbone flexibility}

We have measured $\left\{{ }^{1} \mathrm{H}\right\}-{ }^{15} \mathrm{~N}$ heteronuclear NOEs in order to characterize the backbone flexibility of sYuaF (Fig. 3b) (Kay et al. 1989). The ps-ns flexibility perfectly matches the structural organization of this protein domain. Most of the $\beta$-barrel core of $\mathrm{sYuaF}$ shows heteronuclear NOEs between 0.7 and 0.8 indicating high order parameters of a rigid protein structure with the loop regions displaying the lowest and the well-aligned $\dot{\beta}$-strands showing the highest values. The irregular, solvent-exposed loop from $\mathrm{Gly}^{43}$ to Thr ${ }^{47}$ shows markedly decreased $\left\{{ }^{1} \mathrm{H}\right\}-{ }^{15} \mathrm{~N}$ NOEs down to 0.43 in accordance with higher flexibility of this region caused by the lack of stabilizing long-range interactions with the rest of the protein. Heteronuclear NOEs of the C-terminal amino acids break down rapidly starting with residue $\mathrm{Glu}^{82}$.

The $N$-terminal $\alpha$-helix of sYuaF shows an interesting behaviour in that its flexibility almost linearly decreases when moving from the well-ordered $\mathrm{Gly}^{22}$ towards the $\mathrm{N}$-terminus. The linker region between the $\alpha$-helix and the $\beta 1$-strand is rigid with heteronuclear NOEs similar to those found in the $\beta 1-\beta 2-$ and $\beta 4-\beta 5$-hairpin loops. Flexibility comparable to the irregular loop from residue $43-47$ is 

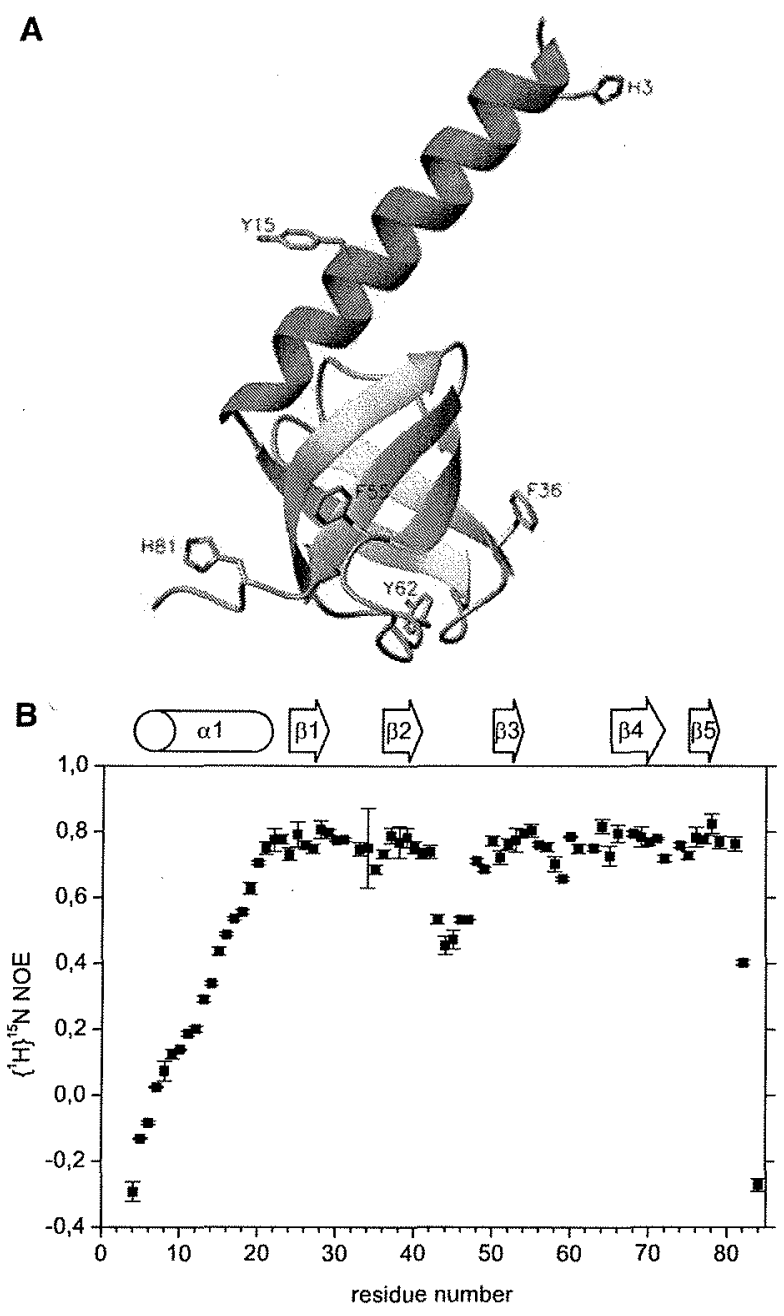

C

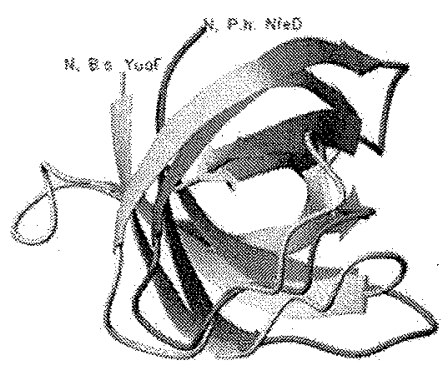

Fig. 3 (a) Location of aromatic amino acids in the 3D structure of sYuaF from $B$. subtilis. Heavy atoms of aromatic side chains are shown in stick representation. All aromatic side chains are solvent exposed. The hydrophobic core of $s$ YuaF's $\beta$-barrel is composed exclusively of aliphatic amino acids. (b) $\left\{{ }^{1} \mathrm{H}\right\}-{ }^{15} \mathrm{~N}$ heteronuclear NOE measured for sYuaF. The intensity ratio between spectra with and without ${ }^{1} \mathrm{H}$ saturation is given as a function of residue number. The cartoon on top of the figure shows the location of secondary structure elements. (c) Superposition of the C-terminal domains of sYuaF from $B$. subtilis (cyan) and the NfeD homolog Ph0471 from P. horikoshii (magenta, PDB code: $2 \mathrm{EXD}$ ). Both proteins display a striking similarity of their $\beta$-sheet cores despite very low homology at the level of primary structure. In contrast to $\mathrm{Y} Y \mathrm{uFF}$, Ph0417 has an unstructured $\mathrm{N}$-terminus. This region has therefore been omitted for clarity reached in the $\mathrm{N}$-terminal $\alpha$-helix at residue $\operatorname{Arg}^{16}$. The orientation of the $\mathrm{N}$-terminal $\alpha$-helix with respect to the $\beta$-barrel core is thus relatively well defined with at least nine strong NOEs between residues $\mathrm{Arg}^{21}, \mathrm{Gly}^{22}$, and $\mathrm{Arg}^{23}$ of the $\mathrm{N}$-terminus and $\mathrm{Ile}^{41}, \mathrm{Leu}^{67}, \mathrm{Val}^{68}$ and $\mathrm{Ile}^{7 !}$ of the core locking the linker and the first two helical residues in position. The $N$-terminal part of the $\alpha$-helix extends away from the protein core. Due to the lack of long-range NOEs the orientation of the $\alpha$-helix is less well defined towards the N-terminus. We find a number of additional NOEs down to residue Glu ${ }^{17}$, however, these are relatively weak suggesting conformational averaging and a decreasing structural definition towards the $\mathrm{N}$-terminus.

\section{Discussion and conclusions}

We report in this article the high-resolution solution structure of YuaF's soluble C-terminal domain, sYuaF, derived from NMR data. In addition, assignments are provided for the first six residues which originate from the MBP-fusion construct used to express and purify sYuaF.

According to our structural analysis, the soluble domain of YuaF from B. subtilis belongs to the diverse class of OB-fold proteins which are most frequently found to be involved in the binding and processing of single-stranded nucleid acids or oligosaccharides (Theobald et al. 2003). Less frequently, OB-fold proteins have been described that take part in protein-protein interactions (Bochkareva et al. 2005).

The structure of YuaF's C-terminus deviates from the canonical OB-fold in several respects: The $\alpha$-helix, usually connecting the $\beta 3$ - and $\beta 4$-strands, is absent in sYuaF. In addition, sYuaF's first $\beta$-strand does not form an extensive anti-parallel $\beta$-sheet with the $\beta 4$-strand resulting in a rather short part of the $\beta$-barrel that is really closed. An interesting extension to the OB-fold is the $N$-terminal $\alpha$-helix of sYuaF that points away from the protein core and potentially forms a more or less rigid spacer between the $\beta$-barrel and the transmembrane part of full-length YuaF. This helix is dominated by polar amino acids. We therefore assume that it is not involved in di- or multimerization of YuaF at the membrane. The sYuaF construct used here does not show any sign of oligomerization, neither from NMR nor from gel filtration.

The "fraying out" of this N-terminal helix in the structural ensemble is a natural consequence of the sole presence of helical NOEs that do not extend beyond residue $i+4$. However, this part of the structure appears to be indeed less stable documented by the reduced $\left\{{ }^{1} \mathrm{H}\right\}-{ }^{15}$ N-NOE and by the broadening of backbone amide signals of $\mathrm{Met}^{4}, \mathrm{Leu}^{5}, \mathrm{Ser}^{7}, \mathrm{Ser}^{8}, \mathrm{Ala}^{9}$, and $\mathrm{Gly}^{22}$ at the beginning 
and the end of sYuaF's $\mathrm{N}$-terminal $\alpha$-helix already at room temperature as observed in the ${ }^{15} \mathrm{~N}$ HSQC spectrum.

The NfeD protein family is a diverse class of proteins attributed with a variety of putative functions (Green et al. 2004). In prokaryotes, SPFH proteins of the reggie/flotillin and stomatin families are almost always found in operon structures together with an NfeD protein, however, there is considerable variability in the amino acid sequences of these NfeD proteins and their function as well as that of their associated SPFH proteins is largely unclear.

Secondary structure predictions suggest that all SPFHassociated $\mathrm{NfeD}$ proteins are composed of $\beta$-structure in their soluble cytoplasmic C-termini, however, only one 3D structure of an SPFH-associated NfeD protein, Ph0471 from the archaeon $P$. horikoshii, has been deposited in the PDB, so far (Kuwahara et al. 2005, PDB code: 2EXD, to be published). We present here the second representative high resolution structure of this region.

With respect to their primary structure, sYuaF and Ph0471 are only distantly related showing a sequence identity of only $21 \%$. Our solution structure of $\mathrm{s} Y$ uaF reveals a striking similarity of the $\beta$-barrel core of both proteins (Fig. $3 \mathrm{c}$ ). The secondary structure matching tool SSM (http://www. ebi.ac.uk/msd-srv/ssm/) reports a $C^{\alpha}$-r.m.s.d of $1.78 \AA$ when fitting 58 residues of $\mathrm{SYuaF}$, from $\mathrm{Glu}^{17}$ to $\mathrm{Ile}^{84}$, with four short gaps onto the structure of Ph0471 (Krissinel and Henrick 2004). In combination with sequence alignments and results from secondary structure predictions this strongly supports our hypothesis that all SPFH-associated NfeD proteins are structurally similar and adopt the OB-fold.

Currently, the biological function of SPFH-associated $\mathrm{NfeD}$ proteins is unknown; however, the finding that SPFH-associated NfeD proteins belong to the OB-fold family may be an important hint on the way to elucidate their function. The vast majority of $\mathrm{OB}$-fold proteins are involved in recognizing single-stranded nucleic acids or oligosaccharides (Theobald et al. 2003). In this context, it is noteworthy that both SPFH-associated NfeD proteins structurally characterized so far, display several aromatic amino acid side chains to the solvent (cf. Fig. 3a). These residues are predestined to be involved in interactions with ligand molecules, either by stacking interactions with nucleobases or by contacting the hydrophobic patches of monosaccharide building blocks. To identify the ligands of these NfeD proteins will be the major objective of further studies and will be key to understanding the functional network of NfeD and SPFH proteins.

Acknowledgements We thank Anke Friemel for help with acquiring spectra on the $600 \mathrm{MHz}$ spectrometer in Konstanz and Dr. Rüdiger Weisemann, Bruker Biospin, for providing instrument time on the $800 \mathrm{MHz}$ spectrometer at Bruker, Rheinstetten. We thank Dr. Jürgen Volz and Klaus Hägele, Nycomed GmbH, for measuring LC-MS spectra of sYuaF and H.J. Dyson and T. Exner for helpful discussion. Financial support of $M$. Hinderhofer through the Transregio Sonderforschungsbereich 11 and $C$. A. Walker through the University of Konstanz is gratefully acknowledged.

\section{References}

Bashford D, Case DA (2000) Generalized born models of macromolecular solvation effects. Annu Rev Phys Chem 51:129-152

Bateman A, Coin L, Durbin R, Finn RD, Hollich V, Griffiths-Iones S, Khanna A, Marshall M, Moxon S, Sonnhammer EL, Studholme DJ, Yeats C, Eddy SR (2004) The Pfam protein families database. Nucleic Acids Res 32:D138-D141

Berjanskii MV, Neal S, Wishart DS (2006) PREDITOR: a web server for predicting protein torsion angle restraints. Nucleic Acids Res 34:W63-W69

Bochkareva E, Kaustov L, Ayed A, Yi GS, Lu Y, Pineda-Lucena A Liao JC, Okorokov AL, Milner J, Arrowsmith CH, Bochkarev A (2005) Single-stranded DNA mimicry in the p53 transactivation domain interaction with replication protein A. Proc Natl Acad Sci USA 102:15412-15417

Case DA, Darden TA, Cheatham TEIII, Simmerling CL. Wang J, Duke RE, Luo R, Merz KM, Wang B, Pearlman DA, Crowley M, Brozell S, Tsui V, Gohlke $H$, Mongan J, Hornak V, Cui G, Beroza P, Schafmeister C, Caldwell JW, Ross WS, Kollman PA (2004) AMBER8. University of Califomia, San Francisco

Delaglio F, Grzesiek S, Vuister GW, Zhu G, Pfeifer J, Bax A (1995) NMRPipe: a multidimensional spectral processing system based on UNIX pipes. J Biomol NMR 6:277-293

Green JB, Fricke B, Chetty MC, von During M, Preston GF, Stewart GW (2004) Eukaryotic and prokaryotic stomatins: the proteolytic link. Blood Cells Mol Dis $32: 411-422$

Güntert P, Mumenthaler C, Wüthrich K (1997) Torsion angle dynamics for NMR structure calculation with the new program DYANA. J Mol Biol 273:283-298

Herrmann T, Güntert P, Wüthrich K (2002) Protein NMR structure determination with automated NOE assignment using the new software CANDID and the torsion angle dynamics algorithm DYANA. J Mol Biol 319:209-227

Kay LE, Torchia DA, Bax A (1989) Backbone dynamics of proteins as studied by ${ }^{15} \mathrm{~N}$ inverse detected heteronuclear NMR spectroscopy: application to staphylococcal nuclease. Biochemistry 28:8972-8979

Keller R (2004) The computer aided resonance assignment tutorial. CANTINA Verlag, Goldau

Koradi R, Billeter M, Wüthrich K (1996) MOLMOL: a program for display and analysis of macromolecular structures. I Mol Graph 14:51-55

Krissinel E, Henrick K (2004) Secondary-structure matching (SSM), a new tool for fast protein structure alignment in three dimensions. Acta Crystallogr D Biol Crystallogr 60:2256-2268

Laskowski RA, Rullmannn JA, MacArthur MW, Kaptein R, Thornton JM (1996) AQUA and PROCHECK-NMR: programs for checking the quality of protein structures solved by NMR. $J$ Biomol NMR 8:477-486

Murzin AG (1993) OB (oligonucleotide/oligosaccharide binding)fold: common structural and functional solution for non-homologous sequences. Embo J 12:861-867

Schubert M, Labudde D, Oschkinat H, Schmieder P (2002) A software tool for the prediction of Xaa-Pro peptide bond conformations in proteins based on $13 \mathrm{C}$ chemical shift statistics. J Biomol NMR 24:149-154

Soto MJ, Zorzano A, Garcia-Rodriguez FM, Mercado-Blanco J, Lopez-Lara IM, Olivares J, Toro $N$ (1994) Identification of a novel Rhizobium meliloti nodulation efficiency nfe gene 
homolog of Agrobacterium ornithine cyclodeaminase. Mol Plant Microbe Interact 7:703-707

Tavernarakis N, Driscoll M, Kyrpides NC (1999) The SPFH domain: implicated in regulating targeted protein turnover in stomatin and other membrane-associated proteins. Trends Biochem Sci $24: 425-427$
Theobald DL, Mitton-Fry RM, Wuttke DS (2003) Nucleic acid recognition by OB-fold proteins. Annu Rev Biophys Biomol Struct 32:115-133

Wiegert T, Homuth G, Versteeg S, Schumann W (2001) Alkaline shock induces the Bacillus subtilis sigma(W) regulon. Mol Microbiol 41:59-71 